\title{
Palaeomagnetic errors related to sample shape and inhomogeneity
}

\author{
Peter Riisager $^{1,2}$ and Niels Abrahamsen \\ ${ }^{1}$ Department of Earth Sciences, Aarhus University, Finlandsgade 8, DK-8200 Aarhus N, Denmark \\ ${ }^{2}$ Danish Lithosphere Centre, Øster Voldgade 10, DK-1350 København K, Denmark
}

(Received August 26, 2002; Revised February 4, 2003; Accepted February 7, 2003)

\begin{abstract}
When determining the remanent magnetization (RM) of palaeomagnetic rock samples, it is assumed that the samples can be approximated by a magnetic dipole. This assumption greatly simplifies the inverse problem of determining the RM from the measured magnetic field of the sample. The magnetic field of the normally used cylindrical rock samples is however not identical to that of a dipole and care must be taken not to introduce any systematic errors. A numerical test of the effect of cylindrical sample shape on the determination of RM for a spinner-type magnetometer is presented. We find that for a spinner magnetometer the non-ideal sample shape has an insignificant effect (less than $0.3^{\circ}$ error), for even the smallest possible sample-sensor distance. Comparing static and spinner-type magnetometers, it is clear that spinner-type magnetometers are less affected by the sample shape. Inhomogeneously magnetized samples are an obvious source of error when determining the RM. We numerically test the error in the determination of RM for the simple case of a laminated cylindrical sample with constant direction, but varying intensity of magnetization between the laminae. For strongly inhomogeneous samples we find an error of $\sim 4^{\circ}$ for typical spinner-type magnetometer and $\sim 10^{\circ}$ for static-type magnetometers.
\end{abstract}

\section{Introduction}

In palaeomagnetic investigations (e.g. Butler, 1992; McElhinny and McFadden, 2000) many kinds of errors are involved in the magnetic results to be used in geophysical or geological interpretations. For instance the mineralmagnetic stability of the remanent magnetization (RM), the tectonical, compactional, stratigraphical or other kind of geological "noise", magnetic refraction due to strong magnetization, instrumental noise, way of statistical treatment, etc.

The basis of all paleomagnetic work, however, is the determination of the RM in rock samples. The RM cannot be measured directly, but must be derived from the intensity and direction of the magnetic field measured outside the sample. Hence it is of fundamental importance, how the "outside" magnetic field is inverted into "inside" RM. Current practice is to simplify this inverse problem by assuming the magnetic field of the paleomagnetic sample to be that of a dipole. This assumption will only be exact if the sample is an ideal uniformly magnetized sphere or ellipsoid, but palaeomagnetic samples are neither spheres nor always uniformly magnetized, so the effect of non-ideal samples on the determination of the RM should be examined.

\section{The Effect of Sample Shape}

Paleomagnetic samples are most often cylinders, as this is a shape easy to cut and orient. Even for a uniformly magnetized cylinder, the magnetic field is however not identical to that of a dipole and this could lead to a systematic error in the determination of RM (Larochelle and Pearce, 1969).

Copy right (C) The Society of Geomagnetism and Earth, Planetary and Space Sciences (SGEPSS); The Seismological Society of Japan; The Volcanological Society of Japan; The Geodetic Society of Japan; The Japanese Society for Planetary Sciences.
To minimize such an error, the length $(\mathrm{L})$ and radius $(\mathrm{R})$ has to be chosen so that the magnetic field of the cylinder has the highest possible resemblance to that of a dipole (Fig. 1). Comparing numerically derived values of the magnetic field along and perpendicular to the axis of a cylindrical sample, it has been shown that the optimum length/radius ratio is $\mathrm{L} / \mathrm{R} \sim 1.7$ (Sharma, 1965, 1968; Larochelle and Pearce, 1969; Collinson, 1983). Directional errors in determination of the RM of rectangular but non-cubical specimens of weekly magnetic sediments were investigated by Steele (1989).

For magnetometers using a field-measuring sensor, the distance (Dist) between the sample and the sensor is another parameter that has to be considered (Fig. 1). This distance has to be big enough so that the non-dipole part of the magnetic field is diminished sufficiently compared to the dipole part. On the other hand it is desirable to get the sensor as close as possible to the sample, to achieve the highest possible sensitivity. Comparing the field along and perpendicular to the axis of a cylinder Collinson (1983) demonstrated that for a static-type magnetometer, measuring the field only along the $x$-, $y$ - and $z$-axes of the cylinder, the error due to sample shape will be $\sim \pm 1^{\circ}$, given that Dist is at least three times the radius $(\mathrm{R})$ of the sample.

A spinner magnetometer measures the field along numerous directions less symmetric than just the $x$-, $y$ - and $z$-axes of the cylinder. To examine the effect of sample shape and finding the optimal sample sensor distance, we numerically simulate the measurement of the RM for a spinner magnetometer. By choosing a magnetization $\left(\mathrm{RM}_{\text {true }}\right)$ of a sample and then determining the best fitting dipole $\left(\mathrm{RM}_{\mathrm{fit}}\right)$ in a fashion similar to that of a spinner magnetometer, the error due to the dipole approximation can be evaluated (compar- 
ing $\mathrm{RM}_{\text {true }}$ and $\left.\mathrm{RM}_{\mathrm{fit}}\right)$. Although the inversion approach differ from the fast Fourier transform algorithm used in some present-day spinner magnetometers, the conclusion reached here is still applicable since simple inversion and FFT will yield the same result for our synthetic data representing the ideal case of noise-free data. The measuring configuration used in the simulations is shown in Fig. 2. The radial component of the magnetic field is calculated at 120 points around the sample in the $y-z(\operatorname{spin} 1), x-z(\operatorname{spin} 2)$ and $x-y(\operatorname{spin} 3)$ planes and the equivalent least square best fitting dipole is found in each of the planes using the method described in Appendix B. The semi analytical method used to calculate the field of the uniformly magnetized cylindrical sample is outlined in Appendix A.

An example of such a simulation is shown in Fig. 3 for a standard cylinder of length $\mathrm{L}=22 \mathrm{~mm}$ and radius $\mathrm{R}=12.5$ $\mathrm{mm}$. The sample-sensor distance Dist $=30 \mathrm{~mm}$ is corresponding to the one currently in use for the FiT Squid spinner in the palaeomagnetic laboratory in Aarhus, and the true

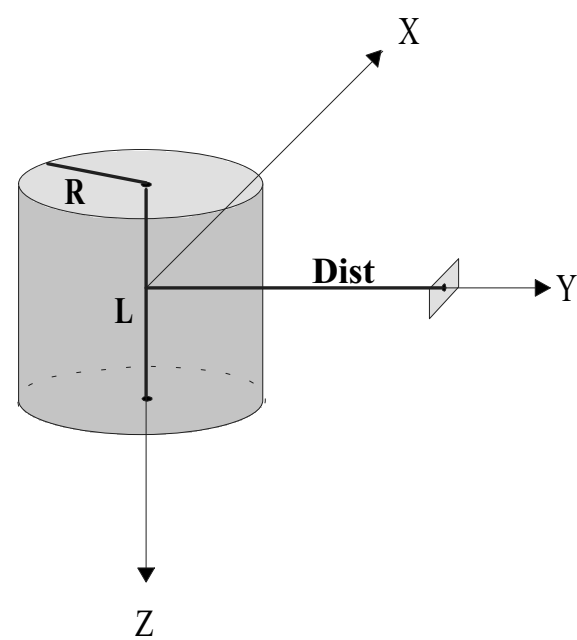

Fig. 1. Cylindrical rock samples in the sample coordinate system. Parameters critical to avoid shape-induced errors are the radius (R) and length (L) of the cylinder and the sample-sensor distance (Dist). magnetization of the cylinder is chosen to give the most severe shape induced error $\left(\mathrm{Dec}_{\text {true }}=0^{\circ}\right.$, Inc $\mathrm{Irue}_{\mathrm{f}}=45^{\circ}$, $\left.\mathrm{M}_{\text {true }}=1 \mathrm{~A} / \mathrm{m}\right)$. Comparing the magnetic field of the cylinder (dots) with that of the equivalent best fitting dipole (solid curve), it is clear that for spin 1 and 2 some difference exists, while for spin 3 the fit is perfect.

By using the dipole inversion (Appendix B), the components of the best fitting dipole are found in each of the planes, and based on these we obtain a magnetization $\left(\right.$ Dec $_{\text {fit }}$, Inc $\left._{\text {fit }}, M_{\text {fit }}\right)=\left(0^{\circ}, 45.12^{\circ}, 0.987 \mathrm{~A} / \mathrm{m}\right)$.

Comparing the true magnetization and that found by our simulated measurement, we see an error in inclination of $\Delta$ Inc $=0.12^{\circ}$ and an intensity $\left(\mathrm{M}_{\mathrm{fit}}\right)$ very close to the intensity of the cylinder $\left(\mathrm{M}_{\text {true }}\right)$. For a homogeneously magnetized cylinder we always find $\operatorname{Dec}_{\text {fit }}=\mathrm{Dec}_{\text {true }}$, independent of the true magnetization.

To test the effect of sample shape for different directions of the true magnetization, the intensity $\left(\mathrm{M}_{\mathrm{fit}}\right)$ as well as the error in inclination $\left(\Delta \mathrm{Inc}=\mathrm{Inc}_{\mathrm{fit}}-\mathrm{Inc}_{\text {true }}\right)$ are found as a function of the true inclination ( Inc $_{\text {true }}$ ). The result is shown in Fig. 4 with $\mathrm{M}_{\text {fit }}$ and Inc plotted against Inc $_{\text {true }}$ for the same measuring configuration as above $(\mathrm{L}=22 \mathrm{~mm}$, $\mathrm{R}=12.5 \mathrm{~mm}$ and Dist $=30 \mathrm{~mm}$ ). We find a maximal $\Delta$ Inc of only $0.12^{\circ}$ and a variation in $\mathrm{M}_{\text {fit }}$ of $0.004 \mathrm{~A} / \mathrm{m}$. The shape induced error for Dist $=30 \mathrm{~mm}$ is hence less than the random error of RM measurements; but it is systematic and in theory Fig. 4 could be used to read the correction needed to remove the effect.

Shortening the sample-sensor distance will increase the sensitivity but also induce a bigger shape induced error. The maximal error in inclination $(\Delta I n c)$ is shown in Fig. 5 for a fixed sample shape $(\mathrm{L}=22 \mathrm{~mm}, \mathrm{R}=12.5 \mathrm{~mm})$, but varying sample-sensor distances. It is remarkable that even for the minimal sample-sensor distance of Dist $=20 \mathrm{~mm}$, we see a maximal $\Delta$ Inc of only $0.28^{\circ}$.

The corresponding error for a static-type magnetometer (e.g. most Squid magnetometers) measuring the field only along the $\pm x$-, $y$ - and $z$-axes of the sample can easily be found. Assuming a dipole, there is a simple linear relationship between $\left(M_{x}, M_{y}, M_{z}\right)$ and $\left(B_{x}, B_{y}, B_{z}\right)$. Reading the
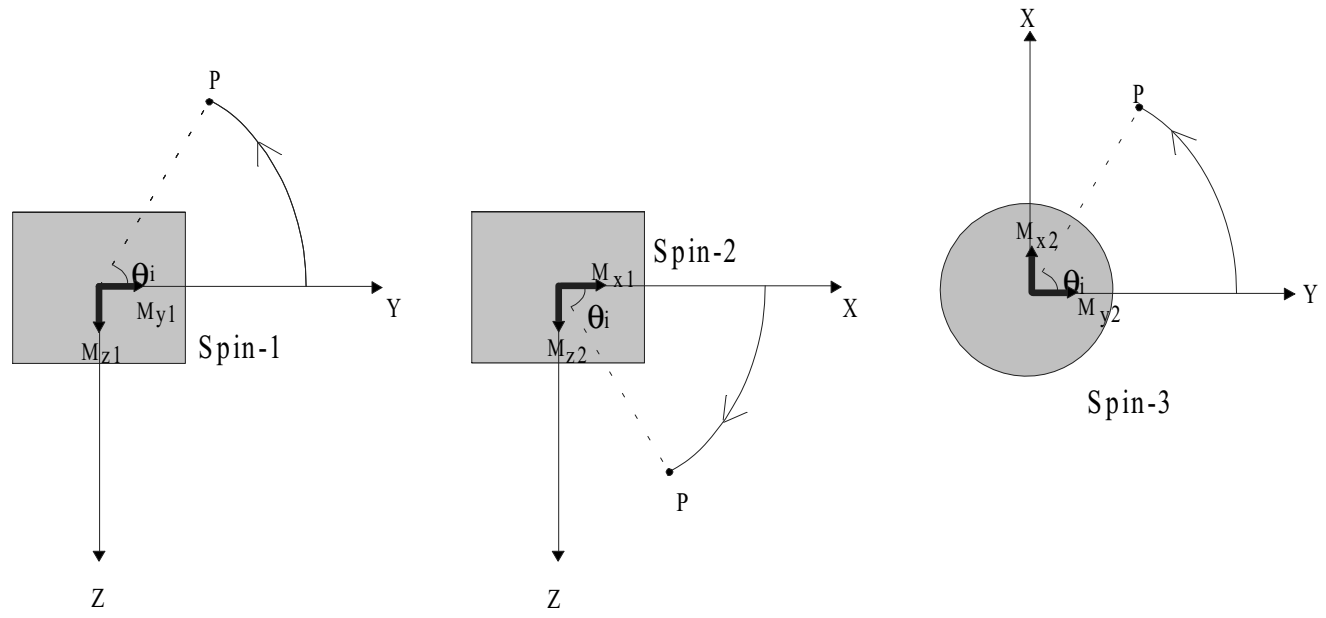

Fig. 2. The 3-spin procedure used for our numerical modeling. For each of the spins, the radial component of the magnetic field is calculated at 120 points (P) around the sample $\left(\theta_{i}=i \cdot 3^{\circ}, i=1,2,3, \ldots, 120\right)$. In each plane the equivalent dipole is found. 


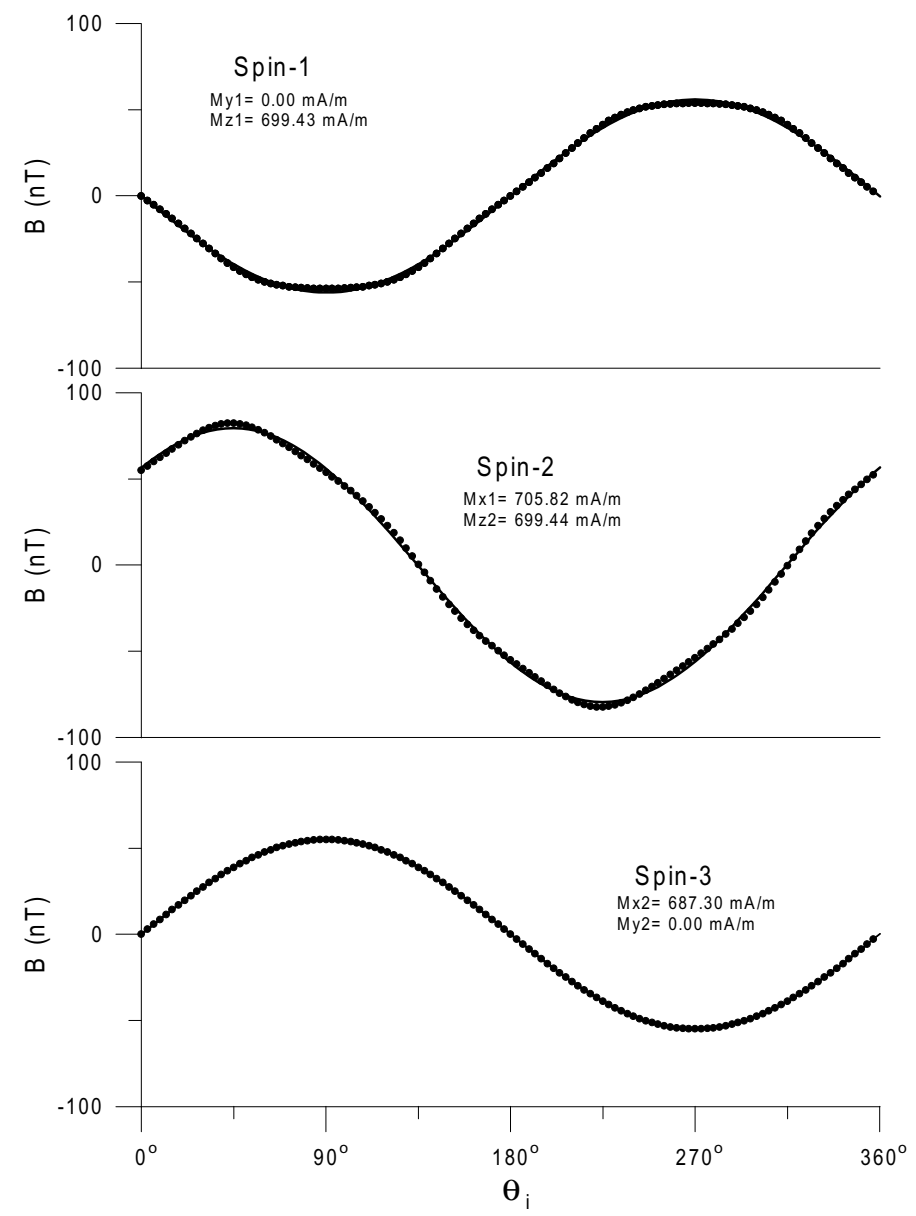

Fig. 3. Example of a numerical simulation with $(\mathrm{L}=22 \mathrm{~mm}, \mathrm{R}=12.5 \mathrm{~mm}$ and Dist $=30 \mathrm{~mm})$ and a magnetization $\mathrm{RM}_{\text {true }}$ of $\left(\mathrm{Dec}_{\text {true }}=0^{\circ}\right.$, Inc true $=45^{\circ}, \mathrm{M}_{\text {true }}=1 \mathrm{~A} / \mathrm{m}$ ). The magnetic field of the cylinder is shown by dots and the best fitting dipole is drawn as a full curve. It is clear that there is some difference between the magnetic field of the cylinder and that of the best fitting dipole, for spin 1 and 2. From the three spin the best fitting dipole is found $\mathrm{RM}_{\text {fit }}$ to be $\left(\operatorname{Dec}_{\mathrm{fit}}=0^{\circ}, \mathrm{Inc}_{\mathrm{fit}}=45.12^{\circ}, \mathrm{M}_{\mathrm{fit}}=0.987 \mathrm{~A} / \mathrm{m}\right)$.

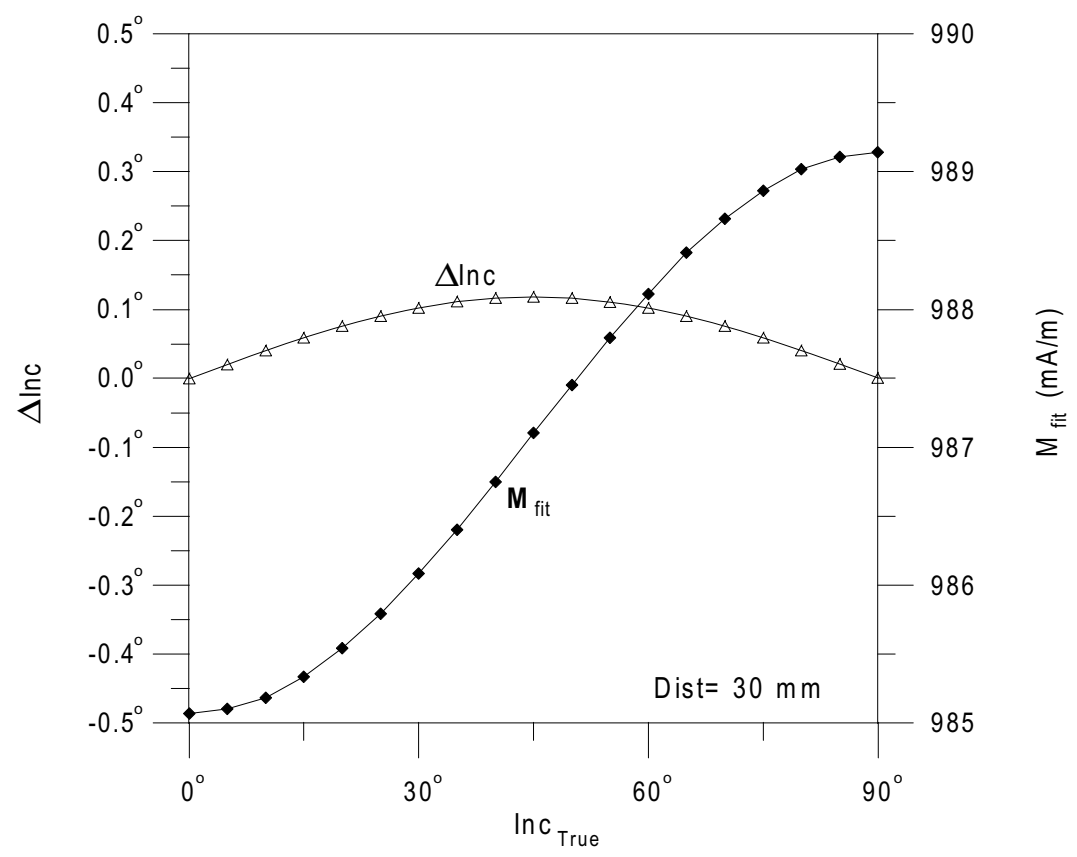

Fig. 4. Intensity of the best-fitting dipole $\left(\mathrm{M}_{\mathrm{fit}}\right)$ and error in inclination $(\Delta \mathrm{Inc})$ as functions of the "true" inclination of the cylindrical sample (Inctrue). Sample-sensor distance is Dist $=30 \mathrm{~mm}$, and $\mathrm{M}_{\text {true }}=1 \mathrm{~A} / \mathrm{m}$. 


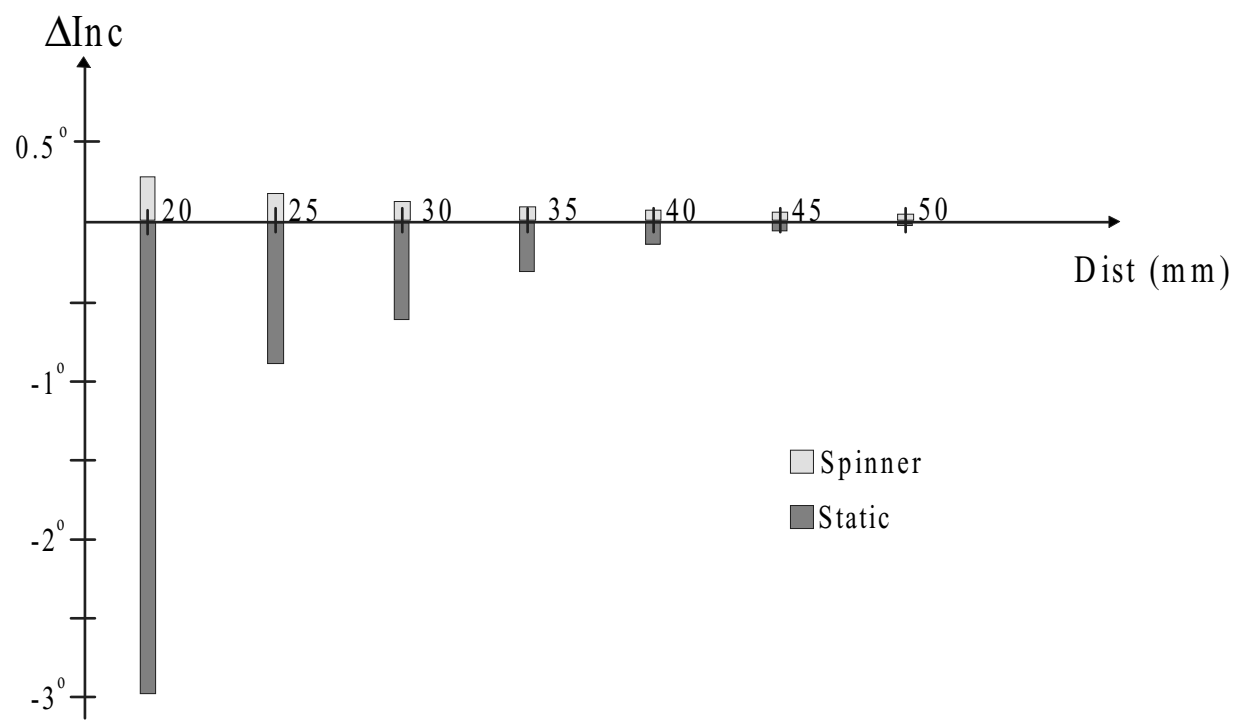

Fig. 5. The maximal error in inclination $(\Delta \mathrm{Inc})$ as a function of sample-sensor distance $($ Dist $)$. The magnetization of the sample is $\left(\mathrm{Dec}_{\text {true }}=0^{\circ}\right.$, Inc $\left._{\text {true }}=45^{\circ}, \mathrm{M}_{\text {true }}=1 \mathrm{~A} / \mathrm{m}\right) . \Delta \mathrm{Inc}$ is given for both a spinner-magnetometer (light-gray columns) and a static-type magnetometer (dark columns).

magnetic field for spin 1 at $\theta=0^{\circ}, 90^{\circ}, 180^{\circ}$ and $270^{\circ}$ gives $B_{y},-B_{z},-B_{y}$ and $B_{z}$, and $\theta=0^{\circ}, 180^{\circ}$ for spin 2 gives $B_{x},-B_{x}$. Using these six readings of the field, $\mathrm{RM}_{\text {true }}$ is found. The error in inclination for a static-type magnetometer is also shown in Fig. 5. Comparing with the values found for a spinner magnetometer we may conclude, that for a given sample-sensor distance, the error due to sampleshape is much smaller for a spinner magnetometer than for a static-type magnetometer.

\section{The Effect of Inhomogeneous Magnetization}

Usually a determination of RM entails multiple measurements of each component of the magnetization allowing a qualitative estimation of the homogeneity. Samples with unacceptable high differences between the multiple measurements can then be rejected, but the significance of inhomogeneity for samples with some milder and acceptable difference is not clear. The unusable chaotic magnetization of slumped/reworked sediments has in some cases indirectly been detected by anisotropy of magnetic susceptibility (Blunk, 1989; Saarinen, 1994). The fact however remains, that there exists no standard procedure in paleomagnetism to directly assess the degree of inhomogeneity.

In some cases it might be desirable to retrieve and use the RM of samples which obviously are inhomogeneous, and before doing so it would be fair to look at the effect inhomogeneity has on the determination of the RM. The effect of inhomogeneity on the determination of RM, has experimentally been tested by building samples with known inhomogeneity and then simply measuring their RM. Such experiments have been performed for different types of magnetometers and a review of the results are given by Collinson (1983). Here we numerically simulate the measurement of annually laminated (varved) sediments, with a markedly banded structure with constant direction but varying intensity of magnetization between the lamellae (Fig. 6). The magnetic field of a laminated sample is calculated by su- perposition of several cylinders (Appendix A) and the effect of the inhomogeniety is examined using the same numerical procedure as described in the previous section.

An example is shown in Fig. 7 for the sample $N=2 a$ (see Fig. 6). The shape of the sample and the sample sensor distance is chosen as in the previous section $(\mathrm{L}=22$ $\mathrm{mm}, \mathrm{R}=12.5 \mathrm{~mm}$ and Dist $=30 \mathrm{~mm}$ ). The dark layer is magnetized $\left(\right.$ Dec $_{\text {true }}=0^{\circ}$, Inc $\left._{\text {true }}=45^{\circ}, \mathrm{M}_{\text {true }}=1 \mathrm{~A} / \mathrm{m}\right)$, while the white layer is non-magnetic. Comparing the magnetic field of the sample (dots) with that of the equivalent best fitting dipole (solid curve) it is clear that a dipole is a very poor approximation to the magnetic field of the sample. The magnetization of the best fitting dipole $\left(\operatorname{Dec}_{\text {fit }}=0^{\circ}\right.$, Inc $\left._{\text {fit }}=45.12^{\circ}, \mathrm{M}_{\mathrm{fit}}=0.4936 \mathrm{~A} / \mathrm{m}\right)$ is however very close to the true magnetization.

The measuring procedure for both static and spinner magnetometers are generally designed to avoid quadrupole components (i.e. an off-centered dipole). Laminated samples with an even number of layers can approximately be represented as samples with an off-centered dipole and we hence find the $\mathrm{RM}_{\text {fit }}$ of the dipole inversion to be close to $\mathrm{RM}_{\text {true }}$ for these samples. Looking at the difference between the best fitting dipole and the field of the $N=2 a$ sample shown in Fig. 7, it is seen that for spin 1 and spin 2 the difference corresponds to a $\sin 2 \theta$ curve (off-centered dipole) and will therefore not have any effect on the best fitting $\sin \theta$ curve (dipole). For spin 3 the difference between the dipole-field and the field of the sample is constant and will neither have any effect.

For an odd number of layers we do however find a difference between $\mathrm{RM}_{\text {true }}$ and that found by the dipole inversion $\left(\mathrm{RM}_{\mathrm{fit}}\right)$. This difference is shown in Fig. 8, with the error in inclination $(\Delta \mathrm{Inc})$ given as a function of the number of layers. The maximal $\Delta \mathrm{Inc}$ is found for sample $N=3 b$ (see Fig. 6) to be ( $\Delta \mathrm{Inc}_{\max }=-3.77^{\circ}$. The corresponding error for a static type magnetometer with the same sample sensor distance shows a maximal $\Delta \mathrm{Inc}$ of $9.59^{\circ}$ for the $N=3 b$ 

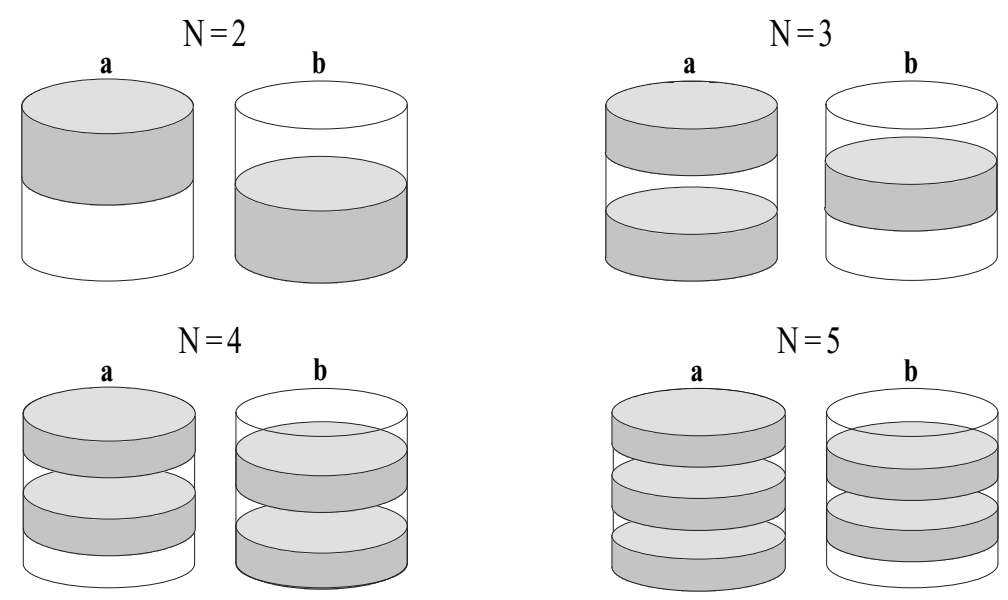

Fig. 6. Laminated cylindrical samples with $\mathrm{N}$ layers. The dark layers are magnetic while transparent layers are non-magnetic.

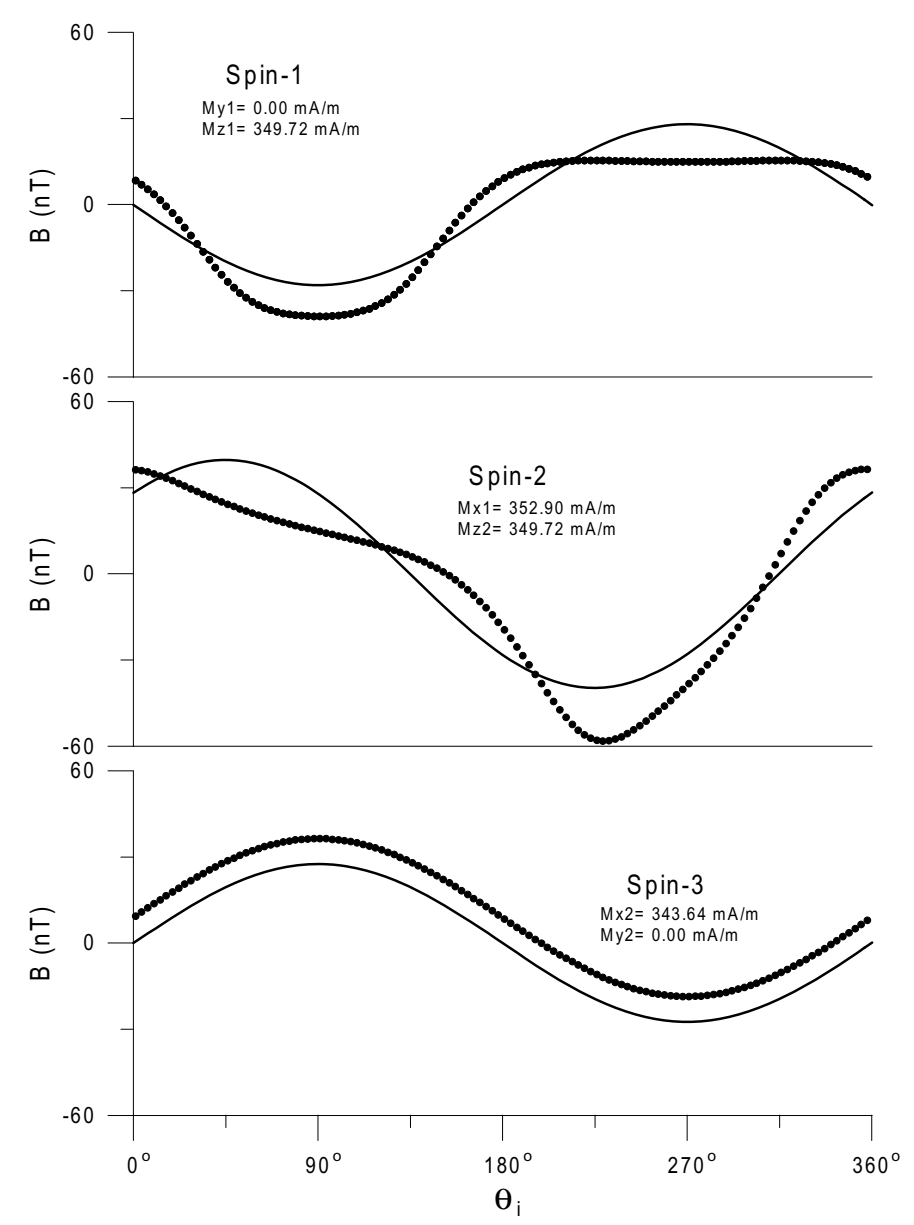

Fig. 7. Example of a numerical simulation of a measurement for a sample with 2 layers, corresponding to sample $N=2 a$ in Fig. 6 . The dark layer is magnetized $\left(\operatorname{Dec}_{\text {true }}=0^{\circ}\right.$, Inc $\left._{\text {true }}=45^{\circ}, \mathrm{M}_{\text {true }}=1 \mathrm{~A} / \mathrm{m}\right)$, while the transparent layer is non-magnetic. The magnetic field of the sample is given as dots and the best fitting dipole is drawn as a full curve. From the three spins the best fitting dipole is found $\mathrm{RM}_{\mathrm{fit}}$ to be $\left(\operatorname{Dec}_{\mathrm{fit}}=0^{\circ}\right.$, Inc $\mathrm{Ifit}=45.12^{\circ}$, $\left.\mathrm{M}_{\mathrm{fit}}=0.4936 \mathrm{~A} / \mathrm{m}\right)$

sample. Comparing the 2 sets of values, it is clear that for a spinner magnetometer the error due to sample-shape is much reduced compared to the effect for a static-type magnetometer.

\section{Discussion and Conclusions}

Before drawing any conclusions on what to expect in a real measuring situation, it is necessary to look at the difference between our numerical modelling and actual measurements of RM. We assume that the field can be measured at a definite point, but a sensor measuring the magnetic field 


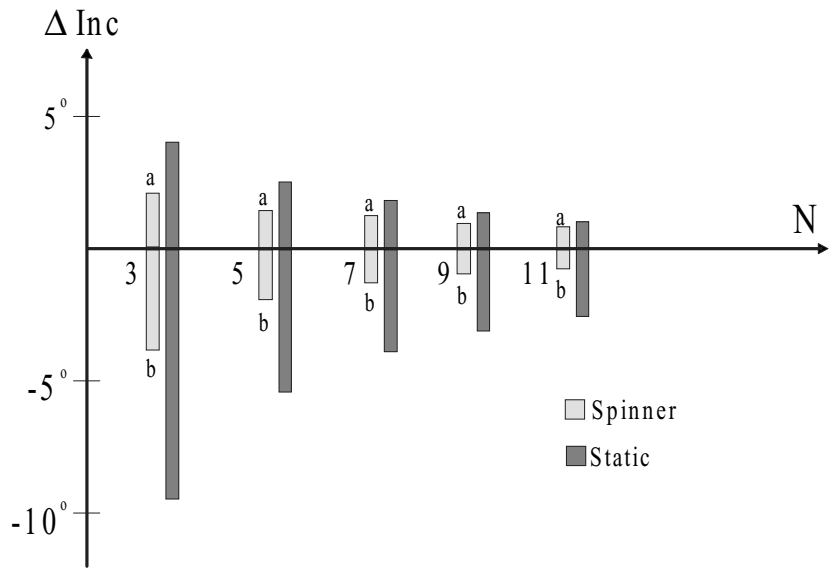

Fig. 8. The error in inclination $(\Delta \mathrm{Inc})$ for laminated samples with $\mathrm{N}$ layers. The sample-sensor distance is $30 \mathrm{~mm}$ and the magnetization of the dark layers is $\left(\mathrm{Dec}_{\text {true }}=0^{\circ}, \mathrm{Inc}_{\text {true }}=45^{\circ}, \mathrm{M}_{\text {true }}=1 \mathrm{~A} / \mathrm{m}\right) . \Delta \mathrm{Inc}$ is given as a function of the number of layers $(\mathrm{N})$ for both a spinner-magnetometer (light-gray columns) and a static-type magnetometer (dark columns).

generally enhances and concentrates the field and this would lead to a smearing of the field not included in our modeling. For cryogenic magnetometers the response-function for field measuring sensors are particularly complex (Parker, 2000; Parker and Gee, 2002) and the real-world situation is obviously more complex than presented here. Also our calculations obviously are free of any noise from sample misorientation or sample-holder magnetization. The measuring procedure we use in our simulations therefore only includes 3 spin for a spinner magnetometer and 6 readings of the field for the static magnetometer. In a real measuring situation the sample is normally spun 3 or 6 times in a spinner magnetometer, and for a static magnetometer the field may be read more than 6 times to reduce the noise.

From the simple simulations it is however clear that: 1) For a given sample sensor distance, the effect of the generally used cylindrical sample shape is much reduced for a spinnertype magnetometer as compared to a static-type magnetometer. 2) For a spinner magnetometer we should expect a shape induced error of only $\pm 0.3^{\circ}$ for the minimal sample-sensor of two times the radius of the sample. 3) For a given sample sensor distance, the effect of inhomogeneous magnetization (Fig. 6) is reduced for a spinner-type magnetometer as compared to a static-type magnetometer.

Besides the error in the direction of RM caused by sample shape, it might also be worthwhile looking at the variation in the intensity $\left(\mathrm{M}_{\mathrm{fit}}\right)$ of the dipole inversion. For all our simulations we find $\mathrm{M}_{\mathrm{fit}}$ to be a little less than the true intensity, but this would just be a question of calibrating the magnetometer. In Fig. 4 we see, however, that $\mathrm{M}_{\mathrm{fit}}$ is varying as a function of the true inclination of the cylinder ( Inc $_{\text {true }}$ ) and for determinations of the anisotropy of remanence, or other research areas where small dependencies of the intensity of magnetization as a function of the direction of magnetization in the sample are interpreted, this could cause problems.

In case of samples with a non-ideal length/radius ratio, it would be simple to avoid any severe shape induced errors by using the method outlined in appendix A to invert the magnetic field into RM. Also for some shape-sensitive experi- ments like the determination of anisotropy of RM it might be of importance to take into account sample shape.

Acknowledgments. We thank Pordur Arason and Randy Enkin for suggestions to improving an early version of the manuscript. Comments and suggestion by the two referees, Jeffrey J. Love and Jeffrey S. Gee, as well as editor Hidefumi Tanaka also improved the manuscript.

\section{Appendix A. Magnetic Field of a Uniformly Magne- tized Cylinder}

The magnetic potential of a uniformly magnetized body can be modeled as the sum of potentials originating from small fictitious magnetic monopoles, distributed over the surface of the body. The differential small monopole $d q$ in the differential small area $d S$ is given by:

$$
d q=\mathbf{J} \cdot \mathbf{n} d S
$$

where $\mathbf{n}$ is the outward unit vector normal to the surface and $\mathbf{J}$ is the uniform magnetization vector of the body. The differential potential $d V(P)$ in point $P$ from the differential small monopole $(d q)$ in point $Q$ is:

$$
d V(P)=\frac{\mu_{o} d q}{4 \pi \mu_{r}|P Q|}, \quad\left(\mu_{r} \text { being unity for air }\right)
$$

To find the magnetic potential in a given point $(P)$ from a magnetic body, we need to integrate the infinitesimal potentials $d V(P)$, originating from the monopoles $d q$, over the entire surface $(S)$ of the body. The magnetic potential $V(P)$ of the body is thus:

$$
\begin{aligned}
V(P) & =\int_{S} d V(P)=\int_{S} \frac{\mu_{o} d q}{4 \pi|P Q|} d S \\
& =\int_{S} \frac{\mu_{o} \mathbf{J} \cdot \mathbf{n}}{4 \pi|P Q|} d S .
\end{aligned}
$$

The magnetic field is given by the gradient of the magnetic potential:

$$
\mathbf{B}(P)=-\nabla V(P) .
$$

The magnetic field of a uniformly magnetized cylinder is found by the linear superposition of two semi-infinite cylinders with opposite magnetization. The surface integral of the semi-infinite cylinders is divided into two surface integrals, one for the circular top (shape 1) and one for the semi-infinite cylinder side (shape 2) (Fig. A1). The two integrals can be solved semi-analytically following the scheme of Gallet and Courtillot (1989).

\section{A.1 Shape 1}

For the circular top surface (shape 1), the distance between the measuring point $P(x, y, z)$ and a magnetic monopole on the surface at $Q(\varepsilon, \eta, 0)$ is:

$$
|P Q|=\sqrt{(x-\varepsilon)^{2}+(y-\eta)^{2}+z^{2}} .
$$

The outward pointing normal to the surface is in the negative $z$-direction, so that:

$$
\mathbf{J} \cdot \mathbf{n}=-J_{z}
$$


b)
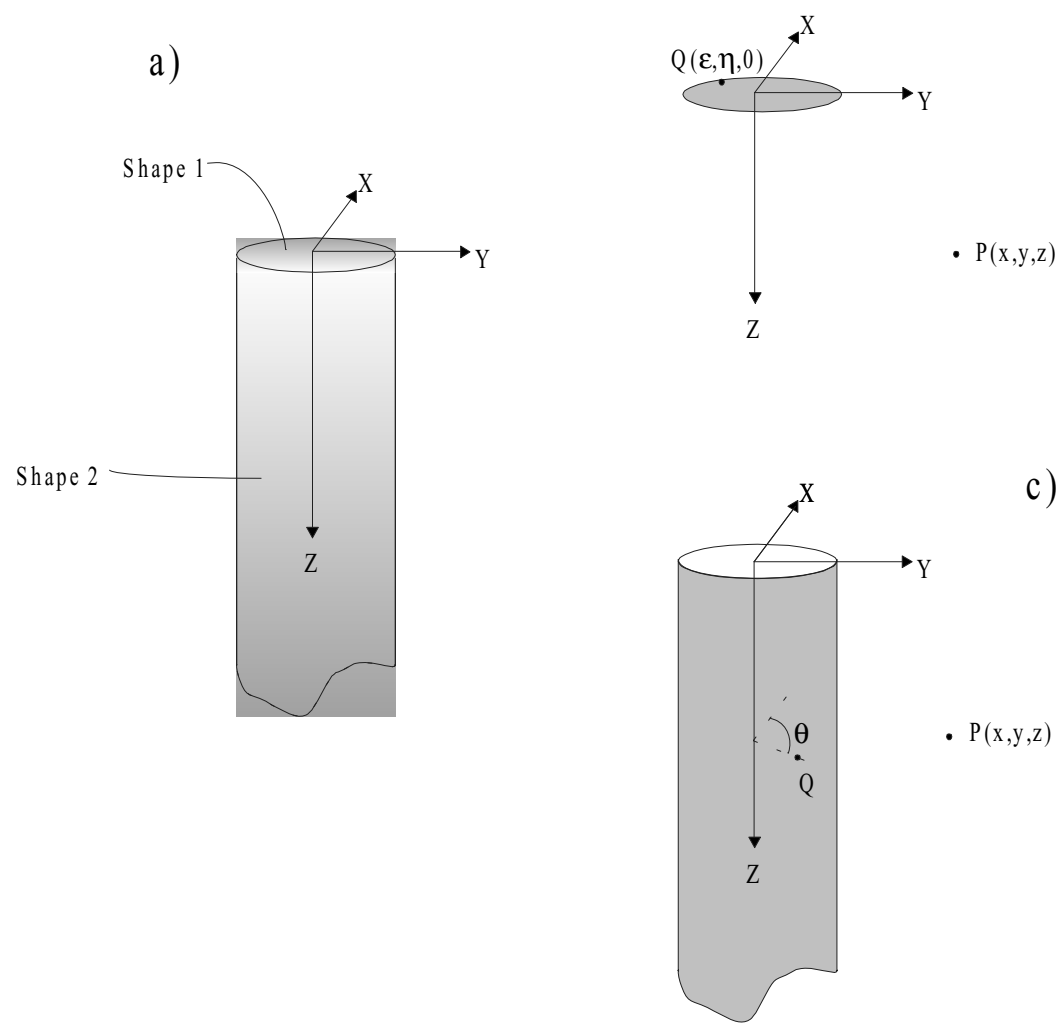

Fig. A1. The surface integral of the semi-infinite cylinder is divided into two surface integrals. One for the top surface (shape 1) and one for the semi-infinite cylinder side (shape 2). The magnetic field at $P(x, y, z)$ is found as the integral field of all the fictitious magnetic monopoles distributed over the surface b) $Q(\varepsilon, \eta, 0)$ of shape 1 and c) $Q(\varepsilon, \eta, \tau)$ of shape 2 .

By integrating along bands parallel to the $y$-axis we get:

$$
\begin{aligned}
V(P) & =\int_{S} \frac{\mu_{o} \mathbf{J} \cdot \mathbf{n}}{4 \pi|P Q|} d S \\
& =\int_{-R}^{R} \int_{-C(\varepsilon)}^{C(\varepsilon)} \frac{d \varepsilon d \eta}{\sqrt{(x-\varepsilon)^{2}+(y-\eta)^{2}+z^{2}}}, \\
C(\varepsilon) & =\sqrt{R^{2}-\varepsilon^{2}}
\end{aligned}
$$

$R$ being the radius of the cylinder.

The magnetic field along the $x$-axis due to shape 1 is found by differentiating the potential with respect to $x$.

$$
\begin{aligned}
B_{x_{1}} & =\frac{-\partial V(P)}{\partial x} \\
& =\frac{-J z \mu_{o}}{4 \pi} \int_{-R}^{R} \int_{-C(\varepsilon)}^{C(\varepsilon)} \frac{(x-\varepsilon) d \varepsilon d \eta}{\left[(x-\varepsilon)^{2}+(y-\eta)^{2}+z^{2}\right]^{3 / 2}} .
\end{aligned}
$$

The integral $K(\varepsilon ; x, y, z)$ can be solved analytically:

$$
\begin{aligned}
K(\varepsilon ; x, y, z) & \\
& =\int_{-C(\varepsilon)}^{C(\varepsilon)} \frac{d \eta}{\left[(x-\varepsilon)^{2}+(y-\eta)^{2}+z^{2}\right]^{3 / 2}} \\
& =\frac{-1}{(x-\varepsilon)^{2}+z^{2}}\left[\frac{y-\eta}{\sqrt{(x-\varepsilon)^{2}+(y-\eta)^{2}+z^{2}}}\right]_{-C(\varepsilon)}^{C(\varepsilon)} \\
& =\frac{1}{(x-\varepsilon)^{2}+z^{2}}\left(\frac{\sqrt{R^{2}-\varepsilon^{2}}-y}{\sqrt{(x-\varepsilon)^{2}+\left(y-\sqrt{R^{2}-\varepsilon^{2}}\right)^{2}+z^{2}}}\right.
\end{aligned}
$$

$$
\left.+\frac{\sqrt{R^{2}-\varepsilon^{2}}+y}{\sqrt{(x-\varepsilon)^{2}+\left(y+\sqrt{R^{2}-\varepsilon^{2}}\right)^{2}+z^{2}}}\right)
$$

Comparing formulas (A.8) and (A.9) we see that $B_{x_{1}}$ can be given as a one-dimensional integral easily solved by any numerical method:

$$
\begin{aligned}
B_{x_{1}} & =\frac{-\partial V(P)}{\partial x} \\
& =\frac{-J_{z} \mu_{o}}{4 \pi} \int_{-R}^{R} K(\varepsilon ; x, y, z)(x-\varepsilon) d \varepsilon .
\end{aligned}
$$

Similarly $B_{y_{1}}$ and $B_{z_{1}}$ can be reduced to one-dimensional integrals of the form:

$$
\begin{aligned}
& B_{y_{1}}=\frac{-\partial V(P)}{\partial y}=\frac{-J_{z} \mu_{o}}{4 \pi} \int_{-R}^{R} L(\varepsilon ; x, y, z) d \varepsilon \\
& B_{z_{1}}=\frac{-\partial V(P)}{\partial z}=\frac{-J_{z} \mu_{o}}{4 \pi} \int_{-R}^{R} K(\varepsilon ; x, y, z) z d \varepsilon .
\end{aligned}
$$

Where $K(\varepsilon ; x, y, z)$ is given in formula (A.9) and $L(\varepsilon ; x, y, z)$ is:

$$
\begin{aligned}
L(\varepsilon ; x, y, z)= & \frac{1}{\sqrt{(x-\varepsilon)^{2}+\left(y-\sqrt{R^{2}-\varepsilon^{2}}\right)^{2}+z^{2}}} \\
& -\frac{1}{\sqrt{(x-\varepsilon)^{2}+\left(y+\sqrt{R^{2}-\varepsilon^{2}}\right)^{2}+z^{2}}} .
\end{aligned}
$$




\section{A.2 Shape 2}

For the lateral side of the cylinder (shape 2) the coordinates of $Q$ and $P$ are $(\varepsilon, \eta, \tau)$ and $(x, y, z)$ respectively (see Fig. A1(c)). Let $\theta$ be the angle between a point and the $x$-axis and $R$ the radius of the cylinder. $\varepsilon$ and $\eta$ can then be written as $\varepsilon=R \cos \theta, \eta=R \sin \theta$ and the distance between $P$ and $Q$ :

$$
\begin{aligned}
|P Q| & =\sqrt{(x-\varepsilon)^{2}+(y-\eta)^{2}+(z-\tau)^{2}} \\
& =\sqrt{(x-R \cos \theta)^{2}+(y-R \sin \theta)^{2}+(z-\tau)^{2}} .
\end{aligned}
$$

The outward pointing normal unit vector is a function of $\theta$ :

$$
\mathbf{J} \cdot \mathbf{n}=\mathbf{J} \cdot \mathbf{n}(\theta)=J_{x} \cos \theta+J_{y} \sin \theta .
$$

From formulas (A.14) and (A.15) we see that the magnetic potential can be written as:

$$
\begin{aligned}
V(P)= & \frac{\mu_{o}}{4 \pi} \int_{0}^{2 \pi} \mathbf{J} \cdot \mathbf{n}(\theta) \int_{0}^{\infty} \\
& \times \frac{R d \tau d \theta}{\sqrt{(x-R \cos \theta)^{2}+(y-R \sin \theta)^{2}+(z-\tau)^{2}}} .
\end{aligned}
$$

Once again the two-dimensional integral for the magnetic potential can be solved semi-analytically. We find the components of the magnetic field expressed by one-dimensional integrals if we split the integrations into cases with magnetization along $x$ and $y$-axes respectively:

$$
\begin{aligned}
B_{x x_{2}} & =\frac{-\partial V\left(J=J_{x}\right)}{\partial x} \\
& =\frac{-J_{x} \mu_{o} R}{4 \pi} \int_{0}^{2 \pi} A(\theta ; x, y, z)(x-R \cos \theta) \cos \theta d \theta
\end{aligned}
$$$$
B_{x y_{2}}=\frac{-\partial V\left(J=J_{y}\right)}{\partial x}
$$$$
=\frac{-J_{y} \mu_{o} R}{4 \pi} \int_{0}^{2 \pi} A(\theta ; x, y, z)(x-R \cos \theta) \sin \theta d \theta
$$$$
B_{y x_{2}}=\frac{-\partial V\left(J=J_{y}\right)}{\partial y}
$$$$
=\frac{-J_{x} \mu_{o} R}{4 \pi} \int_{0}^{2 \pi} A(\theta ; x, y, z)(y-R \sin \theta) \cos \theta d \theta
$$$$
B_{y y_{2}}=\frac{-\partial V\left(J=J_{y}\right)}{\partial y}
$$$$
=\frac{-J_{y} \mu_{o} R}{4 \pi} \int_{0}^{2 \pi} A(\theta ; x, y, z)(y-R \sin \theta) \sin \theta d \theta
$$$$
B_{z x_{2}}=\frac{-\partial V\left(J=J_{x}\right)}{\partial x}
$$$$
=\frac{-J_{x} \mu_{o} R}{4 \pi} \int_{0}^{2 \pi} B(\theta ; x, y, z) \cos \theta d \theta
$$$$
B_{z y_{2}}=\frac{-\partial V\left(J=J_{x}\right)}{\partial z}
$$$$
=\frac{-J_{y} \mu_{o} R}{4 \pi} \int_{0}^{2 \pi} B(\theta ; x, y, z) \sin \theta d \theta
$$

$A(\varepsilon ; x, y, z)$ and $B(\varepsilon ; x, y, z)$ are functions given by:

$$
\begin{aligned}
& A(\theta ; x, y, z) \\
& \quad=\frac{1}{(x-R \cos \theta)^{2}+(y-R \sin \theta)^{2}} \\
& \quad \times\left[1+\frac{z}{\sqrt{(x-R \cos \theta)^{2}+(y-R \sin \theta)^{2}+z^{2}}}\right] \\
& B(\theta ; x, y, z)=\frac{1}{(x-R \cos \theta)^{2}+(y-R \sin \theta)^{2}+z^{2}} .
\end{aligned}
$$

To find the magnetic field of a semi-infinite cylinder, we must add the fields originating from shape 1 and 2 :

$$
\begin{aligned}
B= & \left(B_{x} ; B_{y} ; B_{z}\right) \\
= & \left(B_{x_{1}}+B_{x x_{2}}+B_{x y_{2}} ; B_{y_{1}}+B_{y x_{2}}+B_{y y_{2}} ;\right. \\
& \left.B_{z_{1}}+B_{z x_{2}}+B_{z y_{2}}\right) .
\end{aligned}
$$

To find the magnetic field of a cylinder of finite length we add the fields of two semi-infinte cylinders with opposite magnetization. The numerical integration is performed by the Romberg method (Press et al., 1989).

\section{Appendix B. Finding the RM Using a Dipole Inver- sion}

From the measurement of the radial component of the magnetic field we wish to determine the RM of a sample. Assuming the sample to be a simple dipole, this inverse problem is simple and linear.

We will define a magnetic field pointing away from the sample as a positive field and a field pointing towards the sample as a negative field.

The case, where the field in the $x y$-plane is measured is described in Fig. B1. The radial component of the field, due to $M_{x}$ (remanent magnetisation along the $x$-axis) is:

$$
B_{x}=\frac{2 \mu_{o} V}{4 \pi r^{3}} M_{x} \cos \theta
$$

and the radial field due to $M_{y}$ :

$$
B_{y}=\frac{2 \mu_{o} V}{4 \pi r^{3}} M_{y} \sin \theta
$$

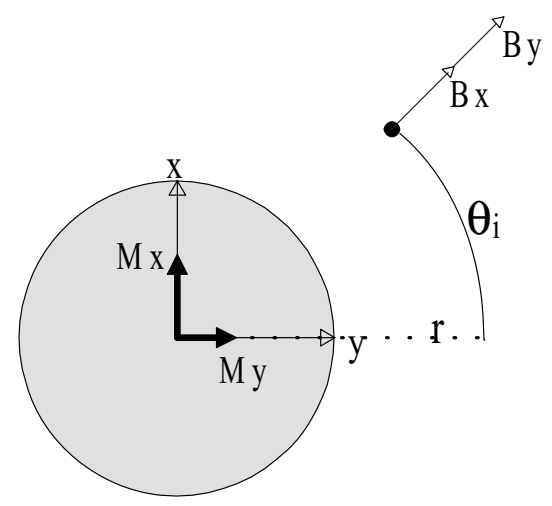

Fig. B1. The radial component of the magnetic field $\left(B_{x}+B_{y}\right)$ is linearly related to the components of magnetization $\left(M_{x}\right.$ and $\left.M_{y}\right)$ for a given $\theta_{i}$ and sample-sensor distance, $r$. 
$V$ is the volume of the sphere, $r$ is the sample-sensor distance and $\theta$ is the angle between the sensor and the $y$-axis (Fig. B1).

The total field for a given angle $\theta_{i}$ is thus:

$$
B_{i}=\frac{2 \mu_{o} V}{4 \pi r^{3}}\left[M_{x} \cos \theta_{i}+M_{y} \sin \theta_{i}\right]
$$

Let us further assume that we measure the field at $N$ points $\left(\theta_{i}=2 \pi i / N, i=1,2, \ldots, N\right)$.

Equation (B.3) can be arranged as a matrix equation of the form $\mathbf{d}=\mathbf{G m}$ :

$$
\left[\begin{array}{c}
B_{1} \\
B_{2} \\
\vdots \\
B_{N}
\end{array}\right]=\left[\begin{array}{cc}
\frac{2 \mu_{o} V}{4 \pi r^{3}} \cos \theta_{1} & \frac{2 \mu_{o} V}{4 \pi r^{3}} \sin \theta_{1} \\
\frac{2 \mu_{o} V}{4 \pi r^{3}} \cos \theta_{2} & \frac{2 \mu_{o} V}{4 \pi r^{3}} \sin \theta_{2} \\
\vdots & \vdots \\
\frac{2 \mu_{o} V}{4 \pi r^{3}} \cos \theta_{N} & \frac{2 \mu_{o} V}{4 \pi r^{3}} \sin \theta_{N}
\end{array}\right] \cdot\left[\begin{array}{c}
M_{x} \\
M_{y}
\end{array}\right] .
$$

The least square inverse solution can now be found as $\mathbf{m}^{\text {est }}=$ $\left[\mathbf{G}^{T} \mathbf{G}\right]^{-1} \mathbf{G}^{T} \mathbf{d}$ (Menke, 1989) and hence $M_{x}$ and $M_{y}$ determined.

\section{References}

Blunk, I., Magnetic susceptibility anisotropy and deformation in Quaternary sediments, Z. Dt. Geol. Ges., 140, 393-403, 1989.

Butler, R. F., Paleomagnetism: Magnetic Domains to Geologic Terranes, 319 pp., Blackwell Scientific Publ., Boston, 1992.
Collinson, D. W., Methods in Rock Magnetism and Palaeomagnetism, 503 pp., Chapman and Hall, London, 1983.

Gallet, Y. and V. Courtillot, Modeling magnetostratigraphy in a borehole, Geophysics, 54, 973-983, 1989.

Larochelle, A. and G. W. Pearce, A possible source of error in determining the remanent magnetization of cylindrical rock specimens with a biastatic magnetometer, Geol. Surv. Can. Pap., 68-62, 1-13, 1969.

McElhinny, M. W. and P. L. McFadden, Paleomagnetism-Continents and Oceans, 382 pp., Academic Press, San Diego, 2000.

Menke, W., Geophysical Data Analysis: Discrete Inverse Theory, 289 pp., Academic Press, San Diego, 1989.

Parker, R. L., Calibration of the pass-through magnetometer-I. Theory, Geophys. J. Int., 142, 371-383, 2000.

Parker, R. L. and J. S. Gee, Calibration of the pass-through magnetometerII. Application, Geophys. J. Int., 150, 140-152, 2002.

Press, W. H., B. P. Flannery, S. A. Teukolsky, and W. T. Vetterling, Numerical Recipes in Pascal, 759 pp., Cambridge University Press, 1989.

Saarinen, T. J., Paleomagnetic study of Holocene sediments of Lake Päijänne (Central Finland) and Lake Paanajärvi (North-West Russia), Geol. Surv. Finland, Bull., 376, 1-87, 1994.

Sharma, P. Vallabh, On the point dipole representation of a Uniformly Magnetized Cylinder, Helv. Phys. Acta, 38, 234-240, 1965.

Sharma, P. Vallabh, Choice of configuration for measurement of magnetic moment of rock specimen with a fluxgate unit, Geoexploration, 6, 101108, 1968.

Steele, W. K., Directional errors in remanent magnetization of non-cubic soft sediment specimens measured with spinner magnetometers, Geophys. J. R. astr. Soc., 96, 333-341, 1989.

P. Riisager (e-mail: pri@dlc.ku.dk) and N. Abrahamsen (e-mail: abraham@geo.au.dk) 